\title{
MASS FLOW IN CLOSE BINARY SYSTEMS
}

\author{
YOJI KONDO \\ Astrophysics Section, NASA Johnson Space Center, Houston, Tex., U.S.A.
}

\author{
G. E. M c C L U S K E Y
}

Division of Astronomy, Dept. of Mathematics, Lehigh University, Bethlehem, Penn., U.S.A.

(Read by F. B. Wood)

\begin{abstract}
The manner of mass flow in close binary systems is examined with a special view to the role of the so-called critical Roche (or Jacobian) lobe taking into consideration relevant physical conditions such as radiation pressure that may affect the restricted three-body problem treatment. The mass does not necessarily flow from component one to component two through the $L_{1}$ point to form a gaseous ring surrounding the latter. These considerations are applied to X-ray binaries with early-type optical components, such as Cyg X-1 (HDE 226868) and 3U 1700 - 37 (HD 153919). In the two bright close binary systems $\beta$ Lyr and UWCMa, which are believed to be undergoing dynamic mass transfer, recent Copernicus Princeton Telescope Spectrometer observations show that the gas giving rise to the prominent ultraviolet emission lines surrounds the entire binary system rather than merely component two. Implications of these observations are also discussed.
\end{abstract}

\section{Introduction}

Recent developments in theory as well as observation of evolutionary processes and mass flow in close binary systems have made it important to examine critically the role of the so-called critical Roche (or Jacobian) lobe. In many instances, it is assumed prima facie that star 1 fills its critical Roche lobe as it evolves and starts transferring mass through the inner Lagrangian point, $L_{1}$, to star 2 . The results of our analysis indicate that this is not necessarily a correct view; in fact, it may be an incorrect view when star 1 is an early-type object.

The physical conditions relevant to the discussion of the Roche lobe have been examined by many investigators and are summarized by Kondo (1974). We briefly review these conditions with some updating. The critical Roche surface exists only when the conditions of the restricted three-body problem are satisfied;i.e., two point masses are in a perfectly circular orbit without any other perturbing forces acting on the third mass-less body. As is discussed in more detail by Kondo (1974), these conditions are not strictly satisfied in real binary systems. If the orbit is eccentric, the critical Roche surface has no mathematical basis. Recently, Shelus (1972) finds that even when the binary orbit is perfectly circular the orbit of the mass-less particle becomes unstable beyond about half way to the $L_{1}$ point from the center of star 1 . Additional complications include the assumption that the two stars can be treated as point masses and the fact that the mean free path of the massless particle is far smaller than the stellar dimensions.

The next section, examines the effect of the radiation pressure, which was recently discussed by Schuerman (1972), as applied to four X-ray binaries with early type components. 


\section{The Effect of Radiation Pressure on the Critical Roche Surface}

Schuerman (1972) has discussed the effects of radiation pressure on the Roche potentials of a close binary system following Kuiper (1941). He concluded that the systems containing a luminous, early-type star do not possess contact surfaces and that mass being lost through the Inner Lagrangian point $\left(L_{1}\right)$ would have access, for a given energy, to a much larger volume surrounding the system than in the case of negligible radiation pressure. The critical Roche lobe also opens up at the far side of component 2 causing $L_{2}$ to disappear.

We are currently making quantitative calculations of the effects of radiation pressure on the size and shape of the Roche surfaces as well as its effects on particle trajectories. We report here some preliminary results as they affect estimates of the physical properties of close binaries containing at least one 0 - or early B-type star; in particular some of the $\mathrm{X}$-ray binaries.

Following Schuerman (1972), the effects of radiation pressure on the zero-velocity surfaces can be calculated through the parameter. $\delta$. In hot stars, we assume the main contribution to the opacity is electron scattering and take $\kappa=0.2(1+X) \simeq 0.35$, where $X$ is the fractional hydrogen abundance by number. Then

$$
\delta=2.68 \times 10^{-5} \frac{L / L_{\odot}}{M / M_{\odot}}
$$

We now estimate the value of this parameter for several X-ray binaries.

The spectral type of the optical component of Cen X-3 has been classified as 09.5B0.5 Ib (Vidal et al., 1974), O9 III-V (Rickard 1974) and O6.5 III (Osmer et al, 1975). As typical values, we may take $M_{0} \sim 20 M_{\odot}, M_{x} \sim 2.5 M_{\odot}$ and $\log L_{0} / L_{\odot}=5.25$ (Mauder 1975), where $M_{0}$ and $L_{0}$ are the mass and luminosity of the optical component and $M_{x}$ is the mass of the X-ray component. The value of $\delta$ is 0.24 .

The optical component in Cyg X-1 has a spectral type of 09.7 Iab and an absolute magnitude of -6.5 (Walborn, 1972, 1973). With a bolometric correction of -3.0 (Allen, 1973), $\log L_{0} / L_{\odot}=5.70$. Hutchings et al. (1973) give $M_{0} \sim 22 M_{\odot}, M_{x} \sim 14 M_{\odot}$. Then $\delta=0.61$.

For $3 \mathrm{U} 1700-37=\mathrm{HD} 153919$, Wolff and Morrison (1974) give the spectral type as O6.5f and estimate $M_{0} \geqslant 25 M_{\odot}$ and $M_{x} \gtrsim 1.4 M_{\odot}$. Hutchings (1975) gives $M_{v}=-6.5$ for the Of star and using a bolometric correction of -3.2 (Morton and Adams, 1968), Log $L_{0} / L_{\odot}=5.78$. If we adopt $M_{0}=30 M_{\odot}$, then $\delta=0.54$.

Morgan, Coude and Whitford (1955) classified HD $77851=3 \mathrm{U} 0900-40$ as B0.5 Ib. Mikkelson and Wallerstein (1974) estimate $M_{0} \sim 22 M_{\odot}, M_{x} \sim 2 M_{\odot}$ and $\log L_{0} / L_{\odot}=5.14$. This gives $\delta=0.17$.

These values of $\delta$ computed for the above binaries yield only the upper bounds on the size of the critical lobes for the optical components since the masses adopted for the optical component should be construed to represent their upper bounds. It is recognized by most workers in the field that component 1 , which evolves first, becomes overluminous after starting the mass flow. Thus these optical components, which are obviously transferring their mass, may have been doing so for an extended period. This is particularly so as we do not have any observational evidence that there were supernova explosions involving these X-ray binaries in the recent past; neutron stars and black-hole are believed to be formed in supernova explosions. 
The results by Schuerman (1972), in particular his Figure 4, show that for each of these four binaries, no contact surface exists. This is quite prominent for Cyg X-1 and HD 153919 where radiation pressure has reduced the effective mass of the optical component by at least a factor of two. The critical Roche surface, figure eight, no longer closes behind the less massive component. Mass leaving the luminous, massive component at the $L_{1}$ point, or from any point on the Roche lobe surrounding this star, can reach a much larger volume surrounding the binary, even for very low initial velocities, than would be the case for negligible radiation pressure.

It is interesting to note that Bessell et al. (1975) have proposed a model, based on $\mathrm{H} \alpha$ observations, of gas outflow in the HD 77581 system just as one would expect from the topological structure of the Roche surfaces in the orbital plane when radiation pressure is large enough to cause the disappearance of the critical Roche surface.

Radiation pressure also reduces the size of the critical Roche lobe surrounding the component which is the source of this pressure. The $L_{1}$ point moves closer to this star. This is of great importance for the X-ray binaries since most investigators assume that the usual Roche lobes, radiation pressure neglected, allow one to surmise some of the physical properties, e.g., the radius of the optical component, of the system if one assumes that the $\mathbf{O}$ or early $\mathrm{B}$ star is in contact with its critical Roche lobe.

The methods discussed by Szebehely (1967), for example, to compute the Roche surfaces and positions of the Lagrangian points are easily modified to take radiation pressure into account if it is independent of position as discussed by Schuerman (1972).

The critical Roche lobe surrounding the hot component which is the source of the radiation pressure shrinks as $\delta$ increases from 0 towards 1 , while the critical lobe surrounding the other component expands. Consequently, use of the conventional critical Roche lobes to estimate values of radii and mass ratios may be completely unwarranted if an $\mathrm{O}$ or B-type star is involved.

Consider the $L_{1}$ point. We adopt the notation by Schuerman (1972) and let $x_{1}(0)$ be the $x$-coordinate of the $L_{1}$ point when $\delta=0$ and $x_{1}(\delta)$ be the $x$-coordinate for some arbitrary $\delta$. The equation for $x_{1}(\delta)$ is:

$$
\begin{aligned}
& \frac{(1-\delta) \mu}{\left(x_{1}+\mu-1\right)^{2}}-\frac{(1-\mu)}{\left(x_{1}+\mu\right)^{2}}+x_{1}=0 \\
& \left(-\mu<x_{1}<1-\mu\right)
\end{aligned}
$$

This is a quintic equation in $x_{1}$ which has only one real positive root. The interesting characteristics of the Roche surfaces for $\delta=0$ have been tabulated, e.g., by Szebehely (1967) and Plavec and Kratochvil (1964).

Table I lists the values of $x_{1}$, the coordinate of $L_{1}$ point, for $\delta=0$ and for the value derived above for the given binary system. Figure 1 shows a qualitative picture of the situation for Cyg X-1. For Cyg X-1, the radius of the critical Roche lobe surrounding the optical component is $25 \%$ larger for $\delta=0$ than for $\delta=0.61$.

We conclude that for X-ray binaries with hot, luminous optical components, especially Cyg X-1 and HD 153919, radiation pressure is very important. The so-called critical Roche surface (the numeral eight) does not exist; the lobe surrounding the optical component is smaller than in the zero radiation pressure case. Naturally these results apply to any close binary containing a hot star. 


\section{TABLE I}

Coordinates of the internal Lagrangian $\left(L_{1}\right)$ point. The more massive star has mass $\mu$ and is located at $x=1-\mu$

\begin{tabular}{lllll}
\hline Binary & $\mu$ & $\delta$ & $x_{1}(0)$ & $x_{1}(\delta)$ \\
\hline Cen X-3 & 0.89 & 0.24 & -0.590 & -0.554 \\
Cyg X-1 & 0.61 & 0.61 & -0.156 & -0.044 \\
HD 77851 & 0.92 & 0.17 & -0.648 & -0.624 \\
HD 153919 & 0.91 & 0.54 & -0.628 & -0.522 \\
\hline
\end{tabular}

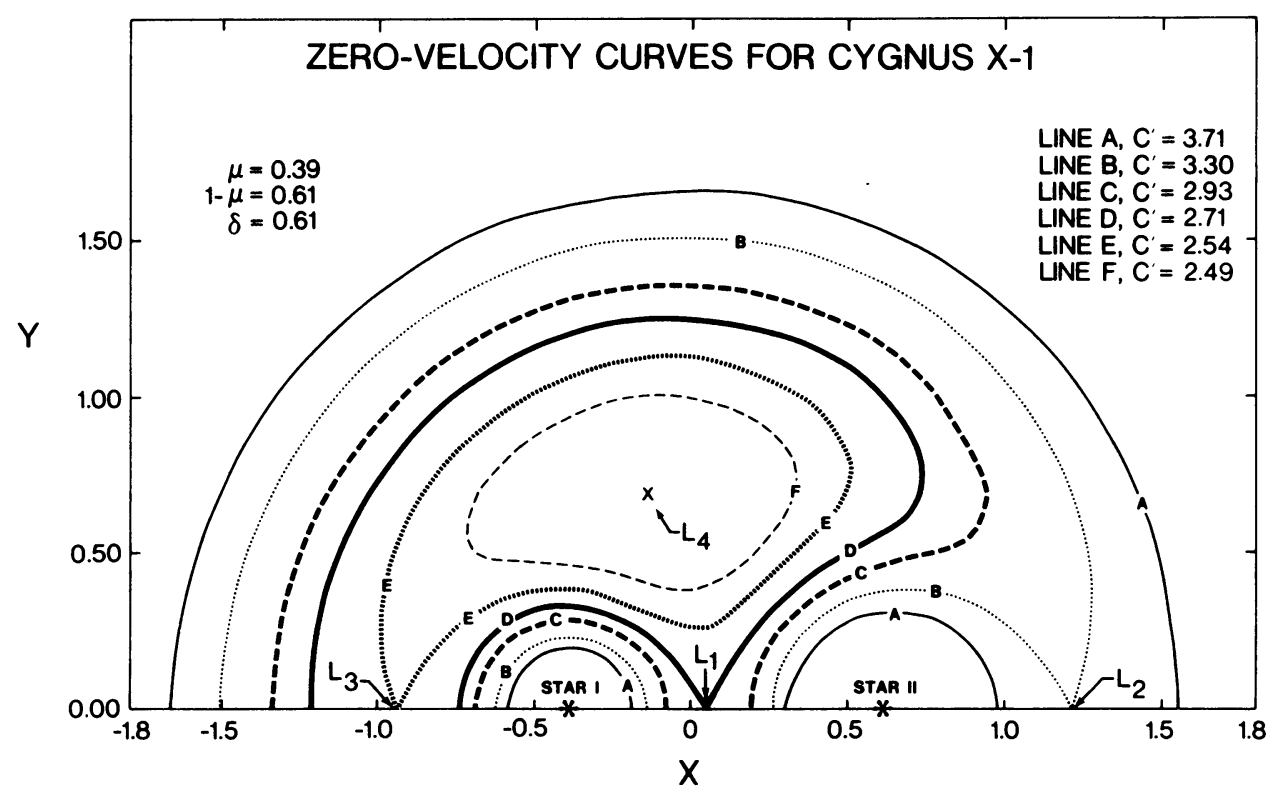

Fig. 1. Critical equipotential surfaces for Cyg X-1.

\section{Observations of Dynamic Mass Flow in the Ultraviolet}

The manner of mass flow is not easy to determine observationally. The difficulty arises in part from the fact that the signature of the mass flow, spectral features associated with the phenomenon, is usually neither distinctive nor prominent. This is because the amount of gas being transferred (either between components 1 and 2 or simply out of component 1 ) is normally relatively small and does not leave a strong spectral signature. Evidence of mass flow may be expected to be more easily detected when the mass being transferred is large such as during the period of dynamic mass transfer. During such a period, which is believed to last only $10^{3} \sim 10^{4} \mathrm{yr}$, as much as $10^{-4}$ to $10^{-6} M_{\odot} \mathrm{yr}^{-1}$ could be leaving component 1 . Although the manner of mass flow is possibly different during the time of dynamic mass transfer, it will provide information on the problem of mass transfer in binaries, on how the gas flows out of component 1 , and/or where the escaping gas ends up. 
The two brightest close binaries that are currently transferring mass dynamically have been observed with the Copernicus Princeton Telescope Spectrometer. The binaries observed are $\beta$ Lyr and UW CMa. Observations of the ultraviolet spectrum of $\beta$ Lyr were obtained by a team of guest investigators, including both of us, and the first results have been reported by Hack et al. (1975). The UW CMa observations were obtained by us in collaboration with Morton and the preliminary results are published (McCluskey et al., 1975). In both of these binaries, prominent emission lines of pluri-ionized atoms are observed in the far ultraviolet region of the spectrum; see Figure 5 on UW CMa and Hack et al. (1975) on $\beta$ Lyr. The radial velocities of these emission lines are basically unchanging indicating that the plasma giving rise to the emissions are stationary with respect to the center of the mass of these binaries. There are additional finer points involving these observations that will not be elaborated upon in this paper. However, the available data for these binaries indicate that the scenario for the transfer of mass from component 1 , filling out the critical Roche lobe, to component 2 through $L_{1}$, in which the flowing mass is simply accreted to component 2 or forms a ring around that star may not be altogether a correct one, not at least for binaries that are transferring mass at a rapid rate.

Note also that the stellar wind is likely to play an important role in mass flow in some stars. For instance, early-type supergiants are believed to be losing mass through such a process. We express our gratitude to F. B. Wood for presenting this paper at the Symposium.

\section{References}

Allen, C. W.: 1973, Astrophysical Quantities, 3rd ed., Athlone, London.

Bessell, M. S., Vidal, N. V., and Wickramasinghe, D. T.: 1975, Astrophys. J. 195, L117.

Hack, M., Hutchings, J. B., Kondo, Y., MoCluskey, G. E., Plavec, M., and Polidan, R. S.: 1975, Astrophys. J. 198, 453.

Hutchings, J. B.: 1975, Astrophys. J. 192, 677.

Hutchings, J. B., Crampton, D., Glaspey, J., and Walker, G. A. H.: 1973, Astrophys. J. 182, 549.

Kondo, Y.: 1974, Astrophys. Space Sci. 27, 293.

Kuiper, G. P.: 1941, Astrophys. J. 93, 133.

Mauder, H.: 1975, Astrophys. J. (Lett.) 195, L27.

McCluskey, G. E., Kondo, Y., and Morton, D. C.: 1975, Astrophys. J. 201, 607.

Mikkelson, D. R. and Wallerstein, G.: 1974, Astrophys. J. 194, 459.

Morgan, W., Code, A., and Whitford, A.: 1955, Astrophys. J. Suppl. 2, 41.

Morton, D. C. and Adams, T. F.: 1968, Astrophys. J. 151, 611.

Osmer, P. S., Hiltner, W. A., and Whelan, J. A. J.: 1975, Astrophys. J. 195, 705.

Plavec, M. and Kratochvil, P.: 1964, Bull. Astr. Inst. Czech. 15, 165.

Rickard, J. J.: 1974, Astrophys. J. 189, L113.

Schuerman, D. W.: 1972, Astrophys. Space Sci. 19, 351.

Shelus, P. J.: 1972, Celes. Mech. 5, 483.

Szebehely, V.: 1967, The Theory of Orbits, Academic Press, New York.

Vidal, N. V., Wickramasinghe, D. T., Peterson, H. A., and Bessell, M. S.: 1974, Astrophys. J. 191 , L23.

Walborn, N. R.: 1972, Astrophys. J. 77, 312.

Walborn, N. R.: 1973, Astrophys. J. 179, L123.

Wolff, S. C. and Morrison, N. D. 1974, Astrophys. J. 187, 69.

\section{DISCUSSION}

Bolton: I have also carried out a calculation for Cyg X-1 using the radiation pressure modified Roche equipotentials. There are three important things that can be seen in such calculations.

(1) Unless the parameter $\delta$ is very large the mass estimates based on Roche equipotentials will not be affected significantly since the two sets of potentials are nearly homologous until $\delta$ becomes large. 
(2) The radius of the visible star and the character of the mass flow may be severely modified by radiation pressure.

(3) The value of $\delta$ cannot be reliably determined because it is extremely sensitive to $T_{\mathrm{e}}$ and $T_{\mathrm{e}}$ is not known with any reliability.

Note Added in Proof. The calculations in this paper have been carried out assuming that the principal mechanism for the radiation pressure is electron scattering. However, as was pointed out by Lucy and Solomon $(1970$, Astrophys. J. 159, 879) the radiation pressure would be increased significantly if the resonance lines of abundant ions were taken into account. Similarly, the radiation pressure would increase materially if the subordinate lines were also taken into account (Castor, Abbott and Klein: 1975, Astrophys. J. 195, 157). Thus, the values of $\delta$, used in the current work, should probably be construed as the lower bounds. 